\title{
Four-Step Classification of Endoscopic Transpapillary Gallbladder Drainage and the Practical Efficacy of Cholangioscopic Assistance
}

\author{
Michihiro Yoshida, Itaru Naitoh, Kazuki Hayashi, Naruomi Jinno, Yasuki Hori, Makoto Natsume, Akihisa Kato, \\ Kenta Kachi, Go Asano, Naoki Atsuta, Hidenori Sahashi, and Hiromi Kataoka \\ Department of Gastroenterology and Metabolism, Nagoya City University Graduate School of Medical Sciences, Nagoya, Japan
}

\section{Article Info}

Received July 31, 2020

Revised September 5, 2020

Accepted September 17, 2020

Published online January 8, 2021

Corresponding Author

Itaru Naitoh

ORCID https://orcid.org/0000-0001-8342-886X

E-mail inaito@med.nagoya-cu.ac.jp
Background/Aims: Although endoscopic transpapillary gallbladder drainage (ETGBD) has been reported as an alternative procedure for acute cholecystitis, it requires advanced endoscopic techniques. In terms of the certainty of achieving drainage, it remains a challenging procedure. The aim of the current study was to elucidate the practical efficacy of cholangioscopic assistance and to develop a new classification that could be used to evaluate the technical difficulty of ETG$\mathrm{BD}$ and provide a theoretical strategy to apply cholangioscopy appropriately for difficult ETGBD.

Methods: A total of 101 patients undergoing ETGBD were retrospectively studied. The characteristics and technical outcomes of ETGBD with conventional ETGBD (C-ETGBD) and SpyGlass DS-assisted ETGBD (SG-ETGBD) were evaluated. The characteristics and technique-dependent factors of unsuccessful C-ETGBD/SG-ETGBD were evaluated using the classification based on the steps of the procedure. The predictive factors of successful C-ETGBD/SG-ETGBD were examined.

Results: C-ETGBD was successful in 73 patients (72.3\%). SG-ETGBD was successful in 11 of 13 patients $(84.6 \%)$ who had C-ETGBD failure. Optional SG-ETGBD significantly increased the final success rate $(94.1 \%)$ compared to C-ETGBD alone $(p=0.003)$. ETGBD procedures could be classified into four steps. SG-assistance worked as an excellent troubleshooter in step 1 (failure to identify the cystic duct orifice) and step 2 (failure of guidewire advancement across the downturned angle of cystic duct takeoff). Magnetic resonance cholangiopancreatography could provide predictive information based on the classification.

Conclusions: Optional SG-ETGBD achieved a significantly higher success rate than C-ETGBD alone. Step classification is helpful for determining the technical difficulty of ETGBD and developing a theoretical strategy to apply cholangioscopy in a coordinated manner. (Gut Liver 2021;15:476-485)

Key Words: Acute cholecystitis; Drainage; Endoscopic retrograde cholangiopancreatography

\section{INTRODUCTION}

Acute cholecystitis (AC) is one of the most common diseases encountered by gastroenterologists. Traditionally, surgical resection, cholecystectomy, has been the standard treatment for AC. ${ }^{1,2}$ But, in patients who are not candidates for surgery, any kind of appropriate drainage might be urgently required to prevent the development of severe complications. Percutaneous transhepatic gallbladder (GB) drainage and aspiration are well-known as the common and traditional techniques that are effective methods for primary drainage..$^{3-8}$ However, these procedures tend to be avoided or cannot be adopted in patients with massive ascites, an anatomically inaccessible GB, a risk of selfremoval of the drainage tube, or a bleeding tendency. ${ }^{9}$

The development of endoscopic retrograde cholangiopancreatography (ERCP) has been spurred by the need for a minimally invasive approach to manage biliary diseases. ${ }^{10}$ Endoscopic transpapillary GB drainage (ETGBD) is currently considered to be the second-line drainage procedure 
for AC. Although ETGBD has been reported to have a significant success rate and a low complication rate, ${ }^{3,11-16}$ this method also has technical difficulties. There may be potential challenges to identifying the cystic duct (CD) on cholangiography and to advancing the guidewire (GW) into the GB through an unfavorable angle of the $\mathrm{CD}, \mathrm{CD}$ tortuosities, or a CD obstruction due to calculus or malignancy. Furthermore, attention should also be paid to ERCP-related adverse events such as post-ERCP pancreatitis.

As an emerging solution to this difficult problem, ETGBD with cholangioscopy-assisted technique has been previously reported. Cholangioscopy could assist in identifying the $\mathrm{CD}$ orifice and simultaneously guide placement of the GW across the CD into the GB (Fig. 1). Few case reports have demonstrated that cholangioscopic assistance is both feasible and effective for GB drainage. ${ }^{17-20}$ In 2006, a single-operator peroral cholangioscopy (SpyGlass; Boston Scientific, Natick, MA, USA) was developed to improve the conventional cholangioscope model, such as increased irrigation flow rate, improved steering, and the capability to perform targeted biopsies under direct visualization. ${ }^{21-23}$ The latest digital version of single-operator peroral cholangioscopy, SpyGlass DS (SG), is currently available to offer additional advantages including sharper image quality and an easier setup process, allowing for both diagnostic and therapeutic benefits. However, it remains unclear how much the success rate of ETGBD could be improved by cholangioscopic assistance. Moreover, which AC patients are the best candidates for cholangioscopic assistance? It is known that SG has a fairly high cost, and it cannot be applied to all cases for AC. Thus, we developed a new theoretical strategy for ETGBD using a new classification based
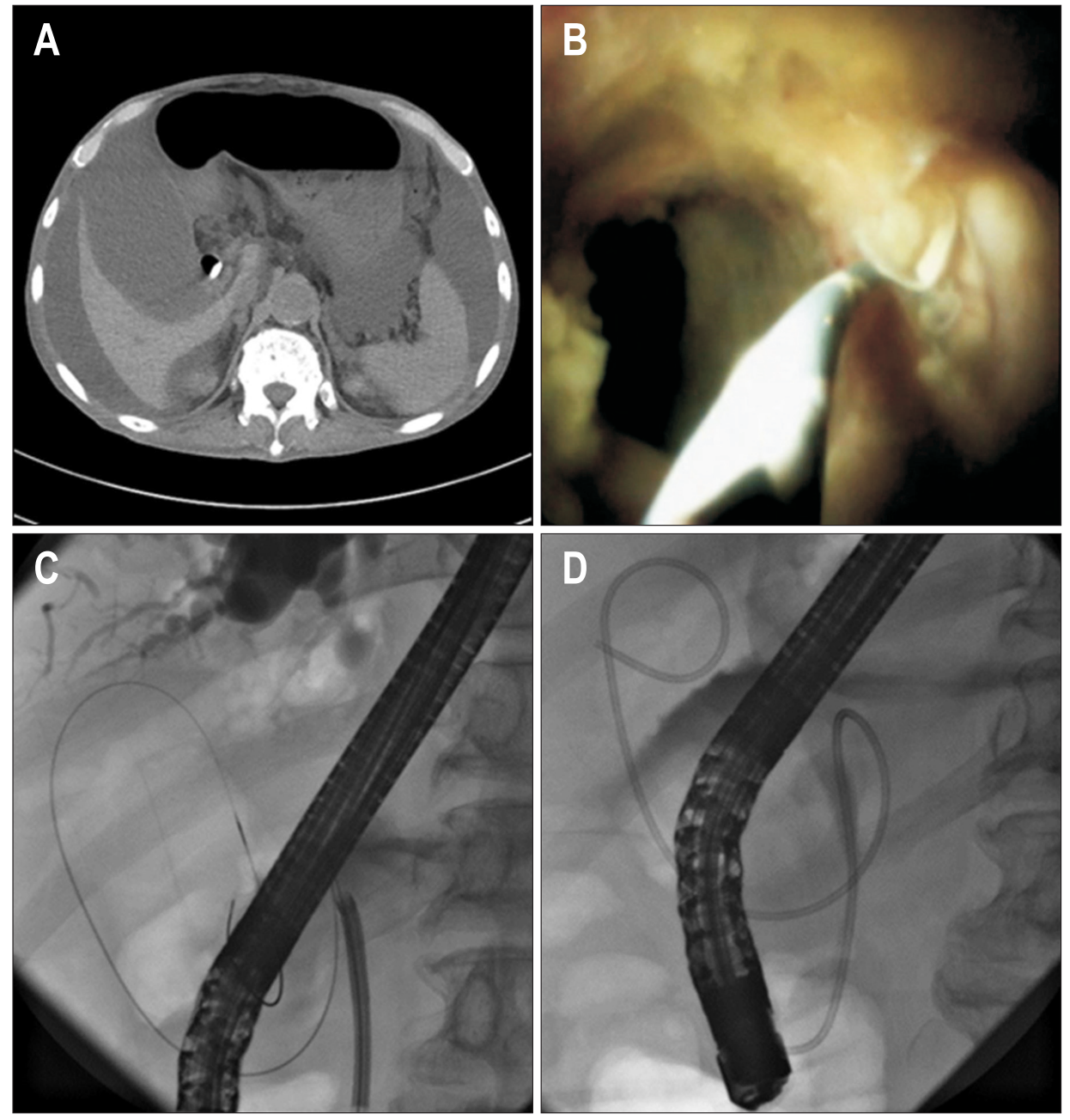

Fig. 1. Endoscopic transpapillary drainage with digital cholangioscopy assistance (SG-ETGBD). (A) Computed tomography shows an enlarged and swollen gallbladder (GB) with a significant amount of ascites. (B) Cholangiography (digital cholangioscopy, SpyGlass DS [SG]) and (C) endoscopic retrograde cholangiopancreatography show a successful endoscopic transpapillary approach into the GB with SG, (D) followed by the insertion of a 7-F naso-GB drainage tube.

SG-ETGBD, SG-endoscopic transpapillary gallbladder drainage. 
on the steps of the procedure. Using this new classification, the aim of this study was to discuss the step-by-step approach for cases of difficult negotiation to the $\mathrm{CD}$, and to elucidate the practical efficacy and feasibility of cholangioscopy.

\section{MATERIALS AND METHODS}

\section{Patients}

A total of 101 consecutive AC patients in whom ETGBD was attempted from April 1, 2008 to June 30, 2020 at Nagoya City University Graduate School of Medical Sciences were retrospectively evaluated. These patients included not only poor surgical candidates, but also those with any reason that would make physicians avoid or hesitate to attempt percutaneous transhepatic GB drainage/aspiration. All patients were classified according to the diagnostic criteria and the severity grading defined in Japanese guidelines, Tokyo Guidelines 2018. "24 "Clinical symptoms" such as right upper quadrant pain and tenderness, "systematic inflammation" such as fever, leukocytosis, and/or high Creactive protein level, and compatible "imaging findings" on ultrasonography or computed tomography, such as thickening of the GB wall or fluid around the GB, were the diagnostic criteria and characteristics. The severity grading was defined using three grades, mild, moderate, and severe.

\section{Procedures of ETGBD and ETGBD combined with cholangioscopy}

Under moderate sedation using a combination of midazolam and pethidine with the patient in the prone position, ERCP using a side-viewing scope was first started with an ERCP catheter and a 0.025-inch GW. After standard biliary cannulation, endoscopic sphincterotomy was performed in cases with a naïve papilla. In cases with comorbid common bile duct (CBD) stones, these stones were removed using baskets or retrieval balloon catheters according to standard techniques. The procedures of conventional ETGBD (C-ETGBD) were then performed, as follows. On cholangiogram, the $\mathrm{CBD}$ was deeply cannulated and injected with contrast to visualize the orifice and the route of the $\mathrm{CD}$. Under fluoroscopic imaging, the GW was manipulated in order to insert it into the GB. This procedure involved the use of standard cannulating catheters, extraction balloons, standard sphincterotomies, and/ or swing tip catheters with the ability to swing in the opposite direction. A number of 0.025 -inch GWs was chosen based on the endoscopist's preference from the followings: VisiGlide2 (Olympus, Tokyo, Japan), RevoWave (PIOLAX
Medical Devices, Yokohama, Japan), Wrangler (PIOLAX Medical Devices), and M-Through (Asahi-Intecc, Seto, Japan). Once the GW could access the GB through the CD, a plastic stent (GB stenting, EGBS) or a 7-F naso-GB drainage (ENGBD) tube was placed into the GB.

In our institution, use of the new digital cholangioscope, "SpyGlass DS" (SG), for cases with unsuccessful CETGBD was started from 2017. In cases of ETGBD with SG-assistance (SG-ETGBD), the SG was inserted through the working channel of the duodenoscope and advanced into the CBD. Direct visualization under SG manipulation could identify the orifice of the $\mathrm{CD}$, and then the GW was inserted across the CD and coiled in the GB. After withdrawal of the SG, the stent or tube was inserted over the GW for EGBS or ENGBD, as in C-ETGBD. Two periods were examined: the earlier (2008-2016) and the later (2017-2020) periods, before and after the introduction of SG for ETGBD assistance, respectively. In the earlier period, all patients underwent C-ETGBD alone (earlier group). In the later period, patients underwent ETGBD that involved the optional choice of subsequent cholangioscopic assistance in cases of C-ETGBD failure (later group).

\section{Definition of the classification based on the steps of ETGBD, "4-Step Classification"}

For the precise evaluation of the procedure, we classified the ETGBD procedures into consecutive steps to identify when the ETGBD attempt failed and was abandoned, as follows: step 0, failure of biliary cannulation; step 1, failure to identify the $\mathrm{CD}$ orifice; step 2, failure to advance the $\mathrm{GW}$ across the $\mathrm{CD}$ takeoff due to an unfavorable angle; step 3a, failure of GW access to the GB due to a CD obstruction (tumor, stone impaction, or inflammation); step $3 b$, failure of GW access to the GB due to multiple tortuosities; and step 4, failure of drainage tube/stent insertion to the GB (Fig. 2).

\section{Study design and outcomes}

First, patient characteristics, technical outcomes, and adverse events of all C-ETGBD were evaluated in this study. Second, patient characteristics, technical outcomes, and adverse events were compared between the earlier and later groups to elucidate the efficacy of optional cholangioscopic assistance. In terms of patient characteristics, age, sex, background characteristics resulting in undergoing ETGBD, AC severity, the presence of GB stones, inflammation, and the condition of papilla were evaluated. The primary outcome was the technical success rate of ETGBD, based on successful placement of the tube or stent into the GB. Adverse events attributed to the performance of ETGBD were defined according to the standard criteria, 


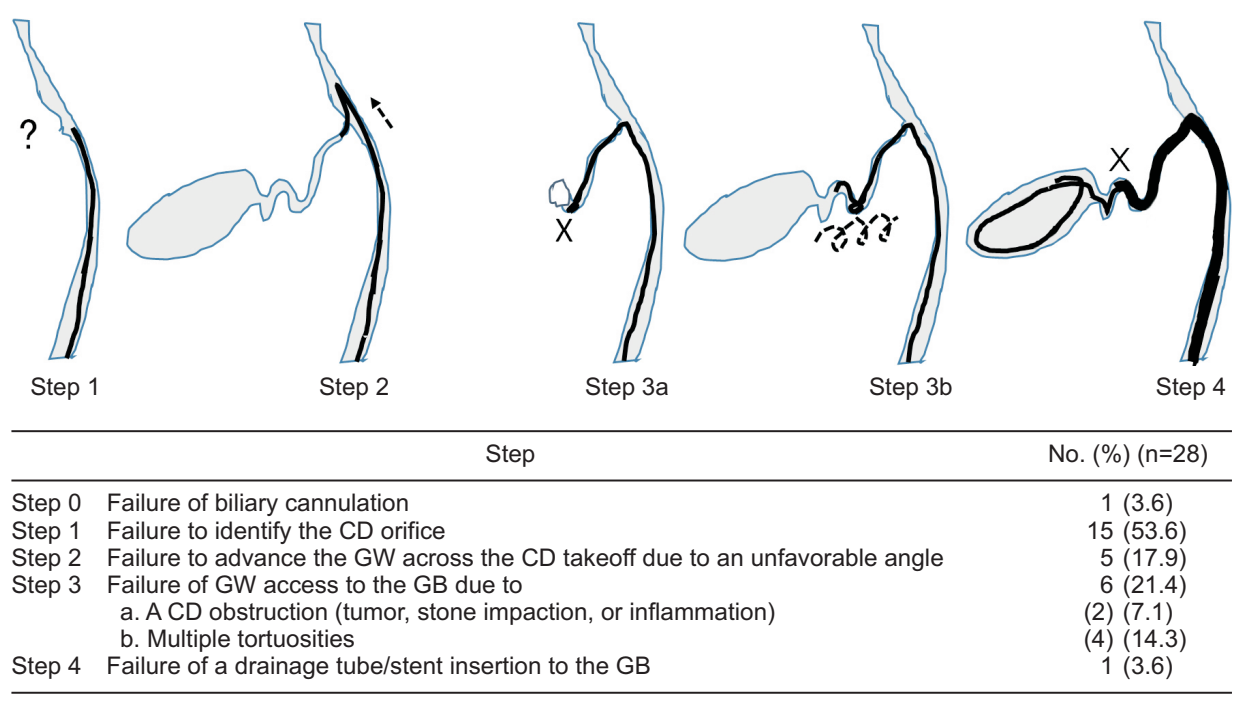

Fig. 2. Four-Step Classification: classification based on the steps of ETGBD. The ETGBD procedure is classified according to the steps at which failure can occur, as follows; step 0 , step 1 , step 2, step 3a, step 3b, and step 4 . ETGBD, endoscopic transpapillary gallbladder drainage; $C D$, cystic duct; GW, guidewire; GB, gallbladder.

Table 1. Patient Characteristics: Success versus Failure (C-ETGBD)

\begin{tabular}{|c|c|c|c|c|}
\hline Characteristics & $2008-2020(n=101)$ & Success ( $n=73$ ) & Failure $(n=28)$ & $\mathrm{p}$-value \\
\hline Age, yr & $77(40-96)$ & $76(40-96)$ & 79 (59-94) & 0.126 \\
\hline Sex, M/F & $64 / 37$ & $45 / 28$ & $19 / 9$ & 0.562 \\
\hline Cholecystitis & & & & 0.118 \\
\hline Mild & $63(62.4)$ & 50 (68.5) & $13(46.4)$ & \\
\hline Moderate & 27 (26.7) & 16 (21.9) & 11 (39.3) & \\
\hline Severe & 11 (10.9) & $7(9.6)$ & 4 (14.3) & \\
\hline WBC, $\times 10^{3} / \mu \mathrm{L}$ & $11.5 \pm 5.50$ & $11.3 \pm 4.92$ & $12.1 \pm 7.02$ & 0.538 \\
\hline $\mathrm{CRP}, \mathrm{mg} / \mathrm{dL}$ & $11.6 \pm 7.91$ & $11.5 \pm 7.91$ & $12.8 \pm 8.17$ & 0.482 \\
\hline GB stone & 76 (75.2) & 54 (74.0) & $22(78.6)$ & 0.632 \\
\hline \multicolumn{5}{|l|}{ Background to challenge ETGBD } \\
\hline Comorbidity with CBD stone & $58(57.4)$ & $42(57.5)$ & $16(57.1)$ & 0.972 \\
\hline Antithrombotic agents & 20 (19.8) & $14(19.2)$ & $6(21.4)$ & 0.799 \\
\hline Comorbidity with suspected GB cancer & $13(12.9)$ & $11(15.1)$ & $2(7.1)$ & 0.287 \\
\hline Dementia & 20 (19.8) & $11(15.1)$ & $9(32.1)$ & 0.064 \\
\hline Ascites & $12(11.9)$ & $8(11.0)$ & $4(14.3)$ & 0.644 \\
\hline Papilla & & & & 0.421 \\
\hline Naïve & $64(63.4)$ & 48 (65.8) & $16(57.1)$ & \\
\hline Post-EST & $37(36.6)$ & 25 (34.2) & $12(42.9)$ & \\
\hline
\end{tabular}

Data are presented as median (range), number (\%), or mean \pm SD.

ETGBD, endoscopic transpapillary gallbladder drainage; C-ETGBD, conventional ETGBD; M, male; F, female; WBC, white blood cell; CRP, C-reactive protein; $\mathrm{GB}$, gallbladder; $\mathrm{CBD}$, common bile duct; $\mathrm{EST}$, endoscopic sphincterotomy.

as follows. Pancreatitis was defined as the onset of new abdominal pain, with at least a 3 -fold elevation of serum amylase or lipase levels, at least 24 hours after the procedure. ${ }^{25}$ Perforation was defined as retroperitoneal or bowelwall perforation, as seen on any image. Hemorrhage was defined as clinical evidence of bleeding, with a decrease in hemoglobin $>2 \mathrm{~g} / \mathrm{dL}$ or the need for endoscopic or transfusion treatment.

To identify the predictive factors of ETGBD difficulty, factors related to the state of the CD to complicate ETGBD were evaluated on the basis of 4-Step Classification described above. Magnetic resonance cholangiopancreatography (MRCP) images examined in advance to ETGBD were also investigated.

This retrospective study was approved by the Institutional Review Board of Nagoya City University Hospital (IRB number: 60-19-0219).

\section{Statistical analysis}

Data were analyzed using the Fisher exact probability test, the $\mathrm{m} \times \mathrm{n}$ test, or the Mann-Whitney U test, as appropriate. p-values $<0.05$ were considered significant. 


\section{RESULTS}

\section{Patients' characteristics}

This study enrolled 101 consecutive patients with AC (median age, 77 years; 64 male and 37 female) who were selected to undergo ETGBD from April 1, 2008 to June 30, 2020 because of the following background characteristics: comorbidity with CBD stones (58 patients, $57.4 \%$ ), antithrombotic therapy (20 patients, 19.8\%), comorbidity with suspected GB cancer (13 patients, 12.9\%), dementia with a possible risk of self-removal of the percutaneous transhepatic GB drainage tube (20 patients, $19.8 \%$ ), or the presence of ascites (12 patients, 11.9\%) (Table 1).

\section{Outcomes of C-ETGBD}

Of the 101 patients, C-ETGBD was successful in 73 patients $(72.3 \%)$. There were no significant differences between the success and failure groups in age, sex, background characteristics resulting in undergoing ETGBD, AC severity, the presence of GB stones, and inflammation.

\section{Comparison of ETGBD before and after the start of cholangioscopic assistance}

The 101 patients were divided into two groups: the first 50 consecutive patients underwent C-ETGBD alone (earlier group), and the next 51 consecutive patients underwent ETGBD with the potential for subsequent cholangioscopic assistance in cases of C-ETGBD failure (later group). As shown in Table 2, there were no significant differences in clinical characteristics between the earlier and later groups. The success rates of C-ETGBD were equivalent (earlier vs later, $72.0 \%$ vs $72.5 \%$, respectively, $\mathrm{p}=0.951$ ). In the later group, 14 patients had C-ETGBD failure, including one patient with CD injury during GW negotiation in whom the procedure was terminated. Thus, 13 patients were candidates to undergo SG-ETGBD, and SG-ETGBD was successful in 11 (84.6\%) of them (Fig. 3A).

The overall success rate was significantly higher in the later group (94.1\%), which involved both C-ETGBD and the optional SG-ETGBD, than in the earlier group that underwent C-ETGBD alone (72.0\%) $(\mathrm{p}=0.003)$ (Fig. 3B).

\section{Adverse events of ETGBD}

$\mathrm{CD}$ injury was the most frequent adverse event in both procedures (C-ETGBD, 5.9\%; SG-ETGBD, 7.7\%), followed by post-ERCP pancreatitis (C-ETGBD, 3.0\%), post-endoscopic sphincterotomy hemorrhage (C-ETGBD, 1.0\%), and peritoneal perforation (C-ETGBD, 1.0\%) (Table 3). All cases with these adverse events recovered with subsequent conservative observation.

\section{The classification based on the steps of ETGBD and predictors of ETGBD difficulty}

To identify the factors that complicate ETGBD, the CETGBD failure group (28 patients, $27.7 \%$ ) was examined. These 28 patients could be classified into four categories according to the consecutive steps that could result in technical failure, as shown in Fig. 2. Of these 28 patients,

Table 2. Patient Characteristics: Earlier Group versus Later Group

\begin{tabular}{|c|c|c|c|}
\hline Characteristics & $\begin{array}{l}\text { Earlier }(2008-2016) \\
\qquad(n=50)\end{array}$ & $\begin{array}{l}\text { Later }(2017-2020) \\
\qquad(n=51)\end{array}$ & p-value \\
\hline Age, yr & $77(40-94)$ & $76(43-96)$ & 0.301 \\
\hline Sex, M/F & $33 / 17$ & $31 / 20$ & 0.586 \\
\hline Cholecystitis & & & 0.561 \\
\hline Mild & 31 (62.0) & 32 (62.7) & \\
\hline Moderate & $12(24.0)$ & 15 (29.4) & \\
\hline Severe & $7(14.0)$ & 4 (7.8) & \\
\hline WBC, $\times 10^{3} / \mu \mathrm{L}$ & $11.1 \pm 5.39$ & $12.2 \pm 5.37$ & 0.202 \\
\hline CRP, mg/dL & $12.6 \pm 8.72$ & $10.7 \pm 6.73$ & 0.337 \\
\hline GB stone & $38(74.0)$ & 38 (74.5) & 0.862 \\
\hline \multicolumn{4}{|l|}{ Background to challenge ETGBD } \\
\hline Comorbidity with CBD stone & $32(64.0)$ & $26(51.0)$ & 0.186 \\
\hline Antithrombotic agents & $9(18.0)$ & $11(21.6)$ & 0.653 \\
\hline Comorbidity with suspected GB cancer & $9(18.0)$ & $4(7.8)$ & 0.128 \\
\hline Dementia & $10(20.0)$ & $10(19.6)$ & 0.961 \\
\hline Ascites & $7(14.0)$ & 5 (9.8) & 0.515 \\
\hline Papilla & & & 0.339 \\
\hline Naïve & $34(68.0)$ & 30 (58.8) & \\
\hline Post-EST & 16 (32.0) & 21 (41.2) & \\
\hline
\end{tabular}

Data are presented as median (range), number (\%), or mean \pm SD.

M, male; F, female; WBC, white blood cell; CRP, C-reactive protein; GB, gallbladder; ETGBD, endoscopic transpapillary gallbladder drainage; CBD, common bile duct; EST, endoscopic sphincterotomy. 

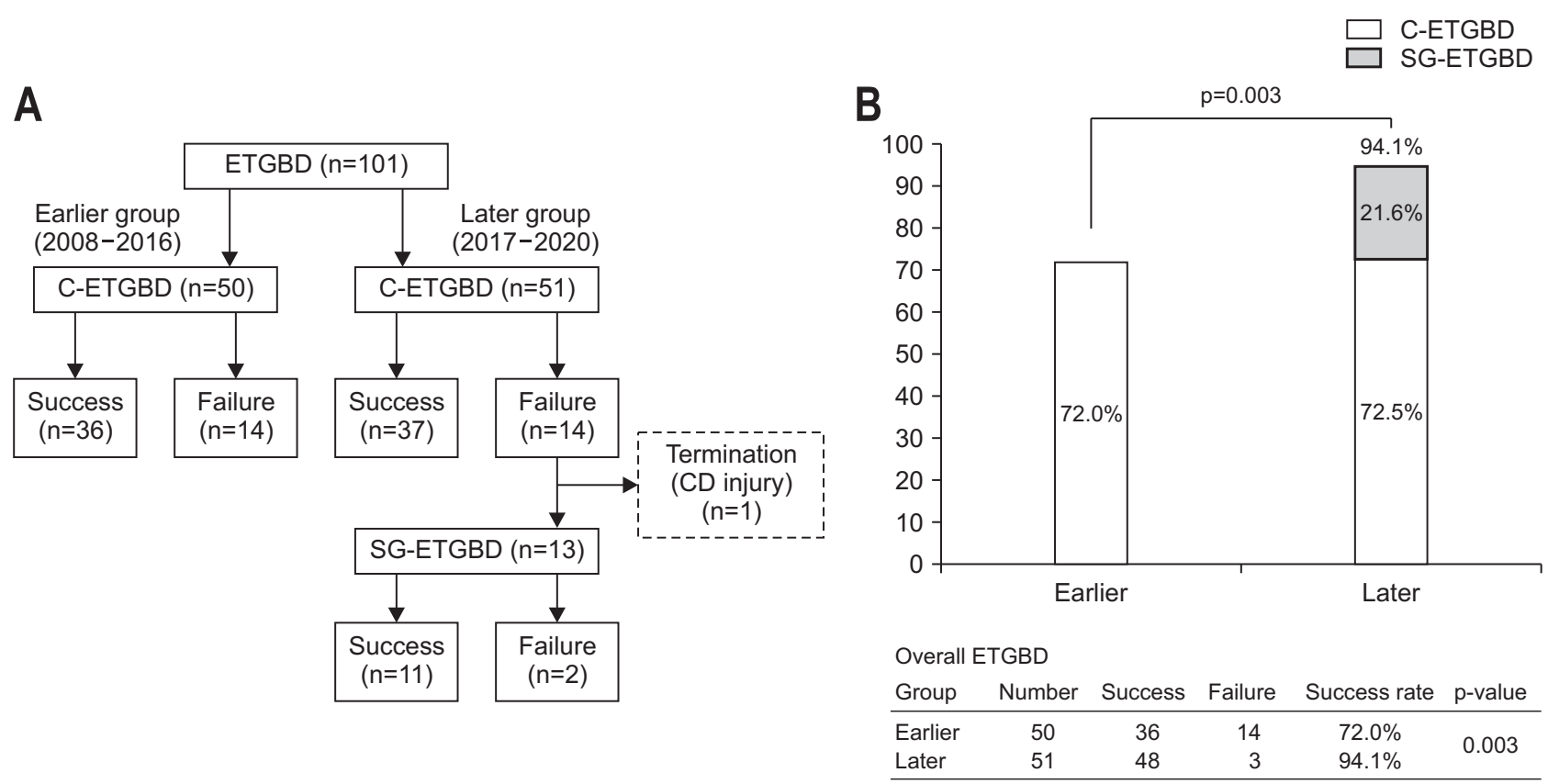

Fig. 3. Clinical courses and overall outcomes of patients with acute cholecystitis who underwent ETGBD. (A) Clinical courses of 101 patients with acute cholecystitis who underwent ETGBD. (B) Overall outcomes of patients who underwent ETGBD.

ETGBD, endoscopic transpapillary gallbladder drainage; C-ETGBD, conventional ETGBD; CD, cystic duct; SG-ETGBD, SpyGlass DS-assisted ETGBD.

Table 3. Complications

\begin{tabular}{lcccc}
\hline \multicolumn{1}{c}{ Complications } & $\begin{array}{c}\text { C-ETGBD } \\
(\mathrm{n}=101)\end{array}$ & $\begin{array}{c}\text { Earlier }(2008-2016) \\
(\mathrm{n}=50)\end{array}$ & $\begin{array}{c}\text { Later (2017-2020) } \\
(\mathrm{n}=51)\end{array}$ & $\begin{array}{c}\text { SG-ETGBD } \\
(\mathrm{n}=13)\end{array}$ \\
\hline CD injury (GW penetration) & $6(5.9)$ & $2(4.0)$ & $4(7.8)$ & $1(7.7)$ \\
Post-ERCP pancreatitis & $3(3.0)$ & $2(4.0)$ & $1(2.0)$ & 0 \\
Post-EST hemorrage & $1(1.0)$ & $1(2.0)$ & & 0 \\
Peritoneal perforation & $1(1.0)$ & $1(2.0)$ & 0 & 0 \\
Total & $11(10.9)$ & $6(12.0)$ & $5(9.8)$ & $1(7.7)$ \\
\hline
\end{tabular}

Data are presented as number (\%).

ETGBD, endoscopic transpapillary gallbladder drainage; C-ETGBD, conventional ETGBD; SG-ETGBD, SpyGlass DS-assisted ETGBD; CD, cystic duct; GW, guidewire; ERCP, endoscopic retrograde cholangiopancreatography; EST, endoscopic sphincterotomy.

15 patients (53.6\%) were categorized as step 1, five patients $(17.9 \%)$ as step 2 , and four patients (14.3\%) as step 3 b, which indicated that whether ETGBD resulted in success depended on several characteristics of the CD. The major steps based on the characteristics of the $\mathrm{CD}$ and the related technique-dependent factors were examined (Table 4). Of 101 patients, one patient, who was categorized as step 0 (failure of biliary cannulation), was excluded for the following analysis $(\mathrm{n}=100)$. In step 1, "failure of identification of the $\mathrm{CD}$ orifice," the critical factor for success was detection of the $\mathrm{CD}$ orifice. As supportive data, the cases with positive CD or GB contrast-filling on cholangiography showed a significantly higher success rate than those that were negative (CD, $81.3 \%$ vs $48.0 \%, p=0.001$; $\mathrm{GB}, 87.2 \%$ vs $63.9 \%, \mathrm{p}=0.011$ ). In step 2 , "failure of GW advancement across the CD takeoff due to an unfavorable angle," the focus was on the direction of the CD takeoff. In terms of the side, left-side takeoff tended to be easier to pass than rightside, but with no significant difference (left vs right, $91.7 \%$ vs $70.5 \%, \mathrm{p}=0.121$ ). In contrast, cases with the $\mathrm{CD}$ takeoff with a downturned angle showed a significantly lower success rate than those with an upward angle (downturned vs upward, $43.8 \%$ vs $78.6 \%$, $\mathrm{p}=0.004)$. In step $3 \mathrm{~b}$, "failure of GW passage to the GB due to multiple tortuosities," the cases with multiple $\mathrm{CD}$ spirals showed a significantly lower success rate (number of CD spirals: $0-1$ vs $\geq 2,82.6 \%$ vs $14.3 \%, \mathrm{p}<0.001)$.

As mentioned above, 13 patients were candidates for SG-ETGBD. Table 5 summarizes the characteristics of these 13 patients focusing on 4-Step Classification and the 
Table 4. Step Classification and the Related Technique-Dependent Factors

\begin{tabular}{|c|c|c|c|c|c|c|c|}
\hline Step & & & All $(n=100)$ & Success ( $n=73$ ) & Failure (n=27) & Success rate, $\%$ & p-value \\
\hline \multicolumn{8}{|l|}{ Step 1-related factor } \\
\hline \multirow[t]{4}{*}{ Contrast filled on cholangiogram } & $C D$ & Yes & 75 (75.0) & $61(83.6)$ & 14 (51.9) & 81.3 & $0.001^{*}$ \\
\hline & & No & $25(25.0)$ & $12(16.4)$ & $13(48.1)$ & 48.0 & \\
\hline & GB & Yes & 39 (39.0) & $34(46.6)$ & 5 (18.5) & 87.2 & $0.011^{*}$ \\
\hline & & No & $61(61.0)$ & $39(53.4)$ & 22 (81.5) & 63.9 & \\
\hline \multicolumn{8}{|l|}{ Step 2-related factor } \\
\hline \multirow[t]{4}{*}{ CD takeoff } & & Right-side & $88(88.0)$ & $62(84.9)$ & 26 (96.3) & 70.5 & 0.121 \\
\hline & & Left-side & $12(12.0)$ & $11(15.1)$ & $1(3.7)$ & 91.7 & \\
\hline & & Upward & $84(84.0)$ & $66(90.4)$ & $18(66.7)$ & 78.6 & $0.004^{*}$ \\
\hline & & Downturned & $16(16.0)$ & $7(9.6)$ & 9 (33.3) & 43.8 & \\
\hline \multicolumn{8}{|l|}{ Step 3b-related factor } \\
\hline Tortuosity & & $0-1$ & $86(86.0)$ & 71 (97.3) & $15(55.6)$ & 82.6 & $<0.001^{*}$ \\
\hline Number of $\mathrm{CD}$ spirals & & $\geq 2$ & $14(14.0)$ & $2(2.7)$ & $12(44.4)$ & 14.3 & \\
\hline
\end{tabular}

Data are presented as number (\%).

$\mathrm{CD}$, cystic duct; $\mathrm{GB}$, gallbladder.

*Statistically significant, $p<0.05$.

Table 5. Characteristics of SG-ETGBD Candidates

\begin{tabular}{|c|c|c|c|c|c|c|c|}
\hline \multirow{2}{*}{ Case } & \multicolumn{2}{|c|}{ Contrast-filled } & \multirow{2}{*}{ CD Takeoff } & \multirow{2}{*}{ No. of CD spirals } & \multirow{2}{*}{ Step of C-ETGBD failure } & \multirow{2}{*}{ SG-ETGBD } & \multirow{2}{*}{ Step of SG-ETGBD failure } \\
\hline & $\mathrm{CD}$ & GB & & & & & \\
\hline 1 & - & - & Down & 0 & Step 1 & Success & \\
\hline 2 & - & - & Up & 0 & Step 1 & Success & \\
\hline 3 & + & - & Down & 1 & Step 2 & Success & \\
\hline 4 & - & - & Down & 2 & Step 1 & Failure & Step 3b \\
\hline 5 & - & - & Down & 2 & Step 2 & Success & \\
\hline 6 & + & - & Down & 2 & Step 2 & Failure & Step 3b \\
\hline 7 & - & - & Up & 0 & Step 1 & Success & \\
\hline 8 & - & - & Up & 1 & Step 1 & Success & \\
\hline 9 & - & - & Up & 0 & Step 1 & Success & \\
\hline 10 & + & - & Up & 2 & Step 3a & Success & \\
\hline 11 & - & - & Up & 0 & Step 1 & Success & \\
\hline 12 & - & - & Up & 0 & Step 1 & Success & \\
\hline 13 & - & - & Down & 2 & Step 2 & Success & \\
\hline
\end{tabular}

CD, cystic duct; GB, gallbladder; ETGBD, endoscopic transpapillary gallbladder drainage; C-ETGBD, conventional ETGBD; SG-ETGBD, SpyGlass DS-assisted ETGBD.

Table 6. Predictors of ETGBD Difficulty: MRCP (n=101)

\begin{tabular}{|c|c|c|c|c|c|}
\hline & All, No. (\%) & No. of success & No. of failure & Success rate, $\%$ & $p$-value \\
\hline MRCP imaging $(n=101)$ & & & & & 0.916 \\
\hline Yes & 75 (74.3) & 54 & 21 & 72.0 & \\
\hline NA & $26(25.7)$ & 19 & 7 & 73.1 & \\
\hline CD on MRCP $(n=75)$ & & & & & $<0.001 *$ \\
\hline Identifiable & $60(80.0)$ & 49 & 11 & 81.7 & \\
\hline Unclear & $15(20.0)$ & 5 & 10 & 33.3 & \\
\hline
\end{tabular}

ETGBD, endoscopic transpapillary gallbladder drainage; MRCP, magnetic resonance cholangiopancreatography; NA, not available; CD, cystic duct.

*Statistically significant, $p<0.05$.

technique-dependent factors. Eight patients had C-ETGBD failure before SG-ETGBD at step 1, four patients at step 2, and one patient at step 3a. SG-ETGBD succeeded in transpapillary GB drainage in $11(84.6 \%)$ of these patients. The remaining two patients had SG-ETGBD failure at step $3 \mathrm{~b}$.
In addition, to identify these predictors of ETGBD difficulty, MRCP was considered. Of all the patients, 75 (74.3\%) were examined with MRCP prior to ETGBD. Although MRCP images or lack thereof did not affect the success rate of ETGBD (yes vs not available, $72.0 \%$ vs $73.1 \%$, $\mathrm{p}=0.916$ ), 
the cases in which the CD could be identified on MRCP showed a significantly higher success rate (identifiable vs unclear, $81.7 \%$ vs $33.3 \%$, p<0.001) (Table 6).

\section{DISCUSSION}

ETGBD has been reported as a novel alternative GB drainage procedure for patients with AC. ${ }^{11-16}$ However, because ETGBD procedures including ENGBD and EGBS require advanced endoscopic techniques, it has not yet been established as a standard procedure. Mohan et al. ${ }^{16}$ recently reported that the pooled technical success rate of ETGBD from multiple databases was $83 \%$. In actual clinical practice, the guideline still notes that ETGBD should be considered in high-volume institutes by skilled pancreatobiliary endoscopists. ${ }^{3}$ Endoscopists must develop methods to provide transpapillary GB drainage with a lower frequency of adverse events. A helpful classification that can predict the difficulty of ETGBD and a reliable ETGBD strategy that overcomes the formidable issues that make ETGBD difficult is very much needed.

As an emerging solution, a handful of case reports have shown the usefulness of cholangioscopy-assisted technique, ${ }^{17-20}$ but no study performed a statistical analysis to investigate it. As shown in Fig. 3A, use of SG resulted in an $84.6 \%$ success rate, and interestingly improved the success rate of C-ETGBD (72.5\%) by $21.6 \%$. This study is the first to demonstrate that ETGBD combined with optional SGETGBD could achieve a significantly higher success rate than C-ETGBD alone (94.1\% vs $72.0 \%, \mathrm{p}=0.003$ ) (Fig. 3B). In terms of adverse events, the addition of SG did not increase the incidence of adverse events (Table 3 ).

The difficulties of ETGBD depend mainly on successful GW access to the GB via the CD. To elucidate these technical difficulties in detail, the steps of ETGBD that failed were evaluated in detail in all 28 failure cases according to a novel classification of procedure steps, the "4Step Classification." As shown in Fig. 2, the first obstacle is to identify the $\mathrm{CD}$ orifice under fluoroscopic observation, which is the most frequent step of failed ETGBD (53.6\%). Inflammation derived from $\mathrm{AC}$ might obscure the $\mathrm{CD}$ orifice, which could hinder passage to the GB. After step 1 , the second potential difficulty is negotiation of the angle of CD takeoff. Left-side takeoff seemed to be easier than right-side $(91.7 \%$ vs $70.5 \%, \mathrm{p}=0.121)$, although the difference was not significant because the left-side cases were in the minority (12.0\%). In most cases, the ERCP cannulation catheter follows the slightly right-curved shape of the distal CBD, and the left-side CD takeoff is sitting on the way of the lateral side of the curved CBD, unlike the right-side
CD takeoff. Cannulation catheters are thus easier to slip in the left-side CD takeoff along with the lateral curve. As expected, cases with a downturned $\mathrm{CD}$ showed lower success rate $(43.8 \%)$. In cases of a downturned $\mathrm{CD}$, the GW tended to deviate from the $\mathrm{CD}$ to the proximal $\mathrm{CBD}$ because of the mechanical characteristics to go upward.

It is noteworthy that SG-ETGBD could overcome these difficulties at step 1 and step 2. As shown in Table 5, cholangioscopy could work as a complete troubleshooter for step 1 problems to identify the orifice of the CD under direct visualization, which was reported and recognized as a good indication of SG-ETGBD in previous case reports. Moreover, our results first showed that cholangioscopy could also work as troubleshooter for step 2 to assist GW advancement into the deeper CD without deviation, because cholangioscopy manipulation could occlude the $\mathrm{CD}$ orifice to block the GW from going upward to the proximal CBD.

In contrast, step $3 \mathrm{~b}$, multiple tortuosities, still remains an issue to be resolved. Because GW negotiation was attenuated through the multiple tortuosities, it became harder to advance the GW to the GB. We have recently reported two useful techniques that might be promising procedures to overcome step 3b: (1) over-the-wire microcatheter manipulation under cholangioscopy-assisted biliary drainage; $^{26}$ and (2) a new easily maneuverable GW. ${ }^{27}$ In the two present cases that failed step $3 \mathrm{~b}$, these new devices were not used.

Moreover, it is also very important to predict whether GW access to the GB will be achieved rapidly or with enormous effort due to the cumbersome steps of ETGBD, as described above. In the present study, the usefulness of MRCP images to predict the difficulties of performing ERCP was demonstrated. Although the degree of inflammation (white blood cell and C-reactive protein) and severity did not affect the success rate (Table 1), an unidentifiable CD on MRCP images was the predictor of ETGBD difficulty (Table 6), which might indicate the local inflammation that caused obstruction of the CD orifice. MRCP images are also helpful to visualize the direction of the $\mathrm{CD}$ takeoff, working as a step 2 predictor. In practice, MRCP could be considered to be performed in selective patients because it is difficult to perform MRCP in patients with poor cooperation or breath holding. Nonetheless, such clues obtained from MRCP images could predict cases of difficult C-ETGBD for which cholangioscopy should be prepared to assist in the performance of ETGBD.

Endoscopic ultrasound-guided GB drainage has been reported as an alternative GB drainage procedure with a higher success rate. ${ }^{16,28-30}$ However, in some particular situations such as pre-surgery malignancy, massive ascites, or 
other high-risk comorbid conditions, the endoscopic transpapillary approach must still be the only choice to achieve GB drainage. Although some case reports have shown that cholangioscopy assistance was a promising choice for successful ETGBD, ${ }^{17-20}$ the present study involving $101 \mathrm{pa}-$ tients, which was a comparatively large sample compared to previous reports, showed the significant efficacy of cholangioscopic assistance to increase the success rate of ETGBD. In addition, the appropriate use of SG should also be considered from the perspective of cost-effectiveness. The strategy of ETGBD along with this 4-Step Classification could help identify the steps causing potential problems and assist in appropriate application of cholangioscopy.

This retrospective study has limitations because of its sample size and the fact that it was a single-center study. Further studies with larger numbers in multicenter prospective settings are needed.

In conclusion, the use of cholangioscopy could achieve significantly higher rates of successful ETGBD. ETGBD procedures could be classified into a series of steps, and the 4-Step Classification is helpful to appreciate the issues that could complicate ETGBD. Cholangioscopic assistance is one useful technique to overcome failure in the early steps of ETGBD. To develop a theoretical strategy and an approach to selecting cases to apply cholangioscopy in a coordinated manner, 4-Step Classification is of significant value.

\section{CONFLICTS OF INTEREST}

No potential conflict of interest relevant to this article was reported.

\section{AUTHOR CONTRIBUTIONS}

Conceptualization of this study: M.Y., I.N. Methodology: M.Y., I.N., K.H. Data curation: M.Y., I.N., K.H., N.J., Y.H., M.N., A.K., K.K., G.A., N.A., H.S., H.K. Data analysis: M.Y., N.J., Y.H., M.N., A.K., K.K., G.A., N.A., H.S., H.K. Project administration: K.H. Supervision: H.K. Writingoriginal draft: M.Y.

\section{ORCID}

Michihiro Yoshida https://orcid.org/0000-0002-4167-2781 Itaru Naitoh https://orcid.org/0000-0001-8342-886X Kazuki Hayashi https://orcid.org/0000-0001-5217-2873 Naruomi Jinno https://orcid.org/0000-0002-2634-2338
Yasuki Hori https://orcid.org/0000-0001-9510-2568 Makoto Natsume https://orcid.org/0000-0002-1716-0583 Akihisa Kato https://orcid.org/0000-0002-7733-7854 Kenta Kachi https://orcid.org/0000-0001-7462-7170 Go Asano https://orcid.org/0000-0002-1528-7572 Naoki Atsuta https://orcid.org/0000-0002-3563-0614 Hidenori Sahashi https://orcid.org/0000-0003-3983-593X Hiromi Kataoka https://orcid.org/0000-0001-9491-0723

\section{REFERENCES}

1. Okamoto K, Suzuki K, Takada T, et al. Tokyo Guidelines 2018: flowchart for the management of acute cholecystitis. J Hepatobiliary Pancreat Sci 2018;25:55-72.

2. Wakabayashi G, Iwashita Y, Hibi T, et al. Tokyo Guidelines 2018: surgical management of acute cholecystitis: safe steps in laparoscopic cholecystectomy for acute cholecystitis (with videos). J Hepatobiliary Pancreat Sci 2018;25:73-86.

3. Mori Y, Itoi T, Baron TH, et al. Tokyo Guidelines 2018: management strategies for gallbladder drainage in patients with acute cholecystitis (with videos). J Hepatobiliary Pancreat Sci 2018;25:87-95.

4. Melin MM, Sarr MG, Bender CE, van Heerden JA. Percutaneous cholecystostomy: a valuable technique in highrisk patients with presumed acute cholecystitis. Br J Surg 1995;82:1274-1277.

5. Kiviniemi H, Mäkelä JT, Autio R, et al. Percutaneous cholecystostomy in acute cholecystitis in high-risk patients: an analysis of 69 patients. Int Surg 1998;83:299-302.

6. Tsutsui K, Uchida N, Hirabayashi S, et al. Usefulness of single and repetitive percutaneous transhepatic gallbladder aspiration for the treatment of acute cholecystitis. J Gastroenterol 2007;42:583-588.

7. Komatsu S, Tsukamoto T, Iwasaki T, et al. Role of percutaneous transhepatic gallbladder aspiration in the early management of acute cholecystitis. J Dig Dis 2014;15:669-675.

8. Komatsu S, Tsuchida S, Tsukamoto T, et al. Current role of percutaneous transhepatic gallbladder aspiration: from palliative to curative management for acute cholecystitis. J Hepatobiliary Pancreat Sci 2016;23:708-714.

9. Itoi T, Sofuni A, Itokawa F, et al. Endoscopic transpapillary gallbladder drainage in patients with acute cholecystitis in whom percutaneous transhepatic approach is contraindicated or anatomically impossible (with video). Gastrointest Endosc 2008;68:455-460.

10. Itoi T, Kawakami H, Katanuma A, et al. Endoscopic nasogallbladder tube or stent placement in acute cholecystitis: a preliminary prospective randomized trial in Japan (with videos). Gastrointest Endosc 2015;81:111-118.

11. Khan MA, Atiq O, Kubiliun N, et al. Efficacy and safety of 
endoscopic gallbladder drainage in acute cholecystitis: is it better than percutaneous gallbladder drainage? Gastrointest Endosc 2017;85:76-87.

12. McCarthy ST, Tujios S, Fontana RJ, et al. Endoscopic transpapillary gallbladder stent placement is safe and effective in high-risk patients without cirrhosis. Dig Dis Sci 2015;60:2516-2522.

13. Mutignani M, Iacopini F, Perri V, et al. Endoscopic gallbladder drainage for acute cholecystitis: technical and clinical results. Endoscopy 2009;41:539-546.

14. Pannala R, Petersen BT, Gostout CJ, Topazian MD, Levy MJ, Baron TH. Endoscopic transpapillary gallbladder drainage: 10-year single center experience. Minerva Gastroenterol Dietol 2008;54:107-113.

15. Kjaer DW, Kruse A, Funch-Jensen P. Endoscopic gallbladder drainage of patients with acute cholecystitis. Endoscopy 2007;39:304-308.

16. Mohan BP, Khan SR, Trakroo S, et al. Endoscopic ultrasound-guided gallbladder drainage, transpapillary drainage, or percutaneous drainage in high risk acute cholecystitis patients: a systematic review and comparative meta-analysis. Endoscopy 2020;52:96-106.

17. Barkay O, Bucksot L, Sherman S. Endoscopic transpapillary gallbladder drainage with the SpyGlass cholangiopancreatoscopy system. Gastrointest Endosc 2009;70:1039-1040.

18. Gutkin E, Hussain SA, Kim SH. The successful treatment of chronic cholecystitis with SpyGlass cholangioscopy-assisted gallbladder drainage and irrigation through self-expandable metal stents. Gut Liver 2012;6:136-138.

19. Shin JU, Lee JK, Kim KM, Lee KH, Lee KT. Endoscopic naso-gallbladder drainage by using cholangioscopy for acute cholecystitis combined with cholangitis or choledocholithiasis (with video). Gastrointest Endosc 2012;76:1052-1055.

20. Tyberg A, Zerbo S, Kahaleh M, Sharaiha RZ. Digital cholangioscopy-assisted gallbladder drainage: seeing is accessing. Endoscopy 2015;47 Suppl 1 UCTN:E417.

21. Chen YK, Parsi MA, Binmoeller KF, et al. Single-operator cholangioscopy in patients requiring evaluation of bile duct disease or therapy of biliary stones (with videos). Gastroin- test Endosc 2011;74:805-814.

22. Navaneethan U, Hasan MK, Lourdusamy V, Njei B, Varadarajulu S, Hawes RH. Single-operator cholangioscopy and targeted biopsies in the diagnosis of indeterminate biliary strictures: a systematic review. Gastrointest Endosc 2015;82:608614.

23. Kurihara T, Yasuda I, Isayama H, et al. Diagnostic and therapeutic single-operator cholangiopancreatoscopy in biliopancreatic diseases: prospective multicenter study in Japan. World J Gastroenterol 2016;22:1891-1901.

24. Yokoe M, Hata J, Takada T, et al. Tokyo Guidelines 2018: diagnostic criteria and severity grading of acute cholecystitis (with videos). J Hepatobiliary Pancreat Sci 2018;25:41-54.

25. Cotton PB, Lehman G, Vennes J, et al. Endoscopic sphincterotomy complications and their management: an attempt at consensus. Gastrointest Endosc 1991;37:383-393.

26. Yoshida M, Kato A, Hayashi K, et al. Novel technique for intraductal cholangioscopy-assisted biliary drainage with over-the-wire microcatheter manipulation. Endoscopy 2019;51:E398-E399.

27. Miyabe K, Kato A, Asano G, et al. Successful endoscopic transpapillary gallbladder stenting using a new easily maneuverable guidewire: a report of two cases. Endoscopy 2019;51:E349-E351.

28. Jang JW, Lee SS, Park DH, Seo DW, Lee SK, Kim MH. Feasibility and safety of EUS-guided transgastric/transduodenal gallbladder drainage with single-step placement of a modified covered self-expandable metal stent in patients unsuitable for cholecystectomy. Gastrointest Endosc 2011;74:176181.

29. Jang JW, Lee SS, Song TJ, et al. Endoscopic ultrasound-guided transmural and percutaneous transhepatic gallbladder drainage are comparable for acute cholecystitis. Gastroenterology 2012;142:805-811.

30. Anderloni A, Buda A, Vieceli F, Khashab MA, Hassan C, Repici A. Endoscopic ultrasound-guided transmural stenting for gallbladder drainage in high-risk patients with acute cholecystitis: a systematic review and pooled analysis. Surg Endosc 2016;30:5200-5208. 